avances

en cienciase ingenierías

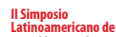
Aplicaciones Nucleares

en la Agricultura

\section{Generación y selección de mutantes de papa (Solanum tuberosum) con resistencia al tizón tardío (Phytophthora infestans)}

\author{
Jorge Rivadeneira ${ }^{1 *}$, Roberto López ${ }^{2}$, María Villavicencio ${ }^{3}$, Xavier Cuesta' \\ 'Instituto Nacional de Investigaciones Agropecuarias (INIAP), Estación Experimental Santa Catalina \\ (EESC), Programa Nacional de Raíces y Tubérculos. Mejía, Ecuador. \\ ¿Universidad Estatal de Bolívar, Facultad de Ciencias Agropecuarias, Recursos Naturales y del \\ Medio Ambiente. Guaranda, Ecuador. \\ ${ }^{3}$ Escuela Politécnica Nacional, Departamento de Ciencias Nucleares, Quito, Ecuador \\ Autor por correspondencia / Corresponding autor, e-mail: jorge.rivadeneira@iniap.gob.ec
}

Editado por /

Edited by:

Gabriela Albán

Recibido /

Received:

28/09/2020

Aceptado /

Accepted:

$13 / 10 / 2020$

Publicado en línea /

Published online:

01/04/2021

\section{Generation and selection of potato mutants (Solanum tuberosum) with resistance to late blight (Phytophthora infestans)}

\section{Resumen}

El objetivo de esta investigación fue generar y seleccionar mutantes con resistencia al tizón tardío, provenientes de la variedad Superchola, mediante radiaciones con fuente ${ }^{60} \mathrm{Co}$. Los ensayos se implementaron en la Estación Experimental Santa Catalina del INIAP, para lo cual, se irradiaron explantes de yemas axilares y apicales de papa in vitro de 45 días de crecimiento. La dosis óptima de irradiación para explantes provenientes de yemas apicales fue de 35 Gy y para explantes de yemas axilares fue de 30 Gy. En laboratorio se realizaron tres micropropagaciones sucesivas para generar y estabilizar las mutaciones. Los mutantes sólidos se inocularon con una suspensión de $0.5 \times 10^{4}$ zoosporas $\mathrm{ml}^{-1}$ de $P$. infestans y se evaluaron ocho días después de la inoculación. En campo se midió la respuesta de 116 mutantes al ataque de tizón tardío. Se incluyeron cinco controles, las variedades resistentes INIAP-Santa Catalina, INIAP-Fripapa y las susceptibles Superchola, INIAP-Gabriela y Uvilla. Se utilizó un diseño experimental de látice parcialmente balanceado 11x11 con tres repeticiones. La prueba de separación de medias utilizada fue Tukey al 5\%. Las variables evaluadas fueron: área bajo la curva de progreso de la enfermedad (ABCPE), calculada con base en las 12 lecturas de severidad y el rendimiento expresado en kilogramos por planta. Los individuos seleccionados como mutantes sólidos correspondieron al 75\% originados de yemas apicales (1319 mutantes) y el 69\% originados de yemas axilares (2145 mutantes). El 80\% de mutantes de origen axilar y el $62 \%$ de origen apical mostraron reacción de susceptibilidad a P. infestans. El porcentaje de mutantes sólidos que mostraron una respuesta de resistencia fue reducido en relación a la población inicial, el 20\% de mutantes de origen axilar y $38 \%$ de mutantes de origen apical mostraron resistencia a P. infestans. En campo para ABCPE se encontraron ocho rangos, las variedades INIAPSanta Catalina, INIAP-Fripapa y los mutantes m67, m92, m39 se ubicaron en los primeros rangos con valores menores a 2536.24 unidades de $A B C P E$. Con respecto al rendimiento se 
establecieron tres rangos, los mutantes m42 y m65 se ubicaron en el primer rango con 1.15 kg planta ${ }^{-1}$. Bajo condiciones de laboratorio se seleccionaron 938 mutantes con resistencia $P$. infestans y 18 en condiciones de campo.

Palabras clave: Explantes, Rendimiento, Severidad, Yemas apicales, Yemas axilares.

\begin{abstract}
To generate and select potato mutants from Superchola potato variety with resistance to late blight, an experiment was implemented at the Santa Catalina Research Station of INIAP. It consisted of ${ }^{60} \mathrm{Co}$ irradiated explants from axillary and apical buds from 45 old-day in vitro potato plants. The optimal irradiation dose for explants from apical buds was $35 \mathrm{~Gy}$ and from axillary buds explants was $30 \mathrm{~Gy}$. Three successive micropropagations were performed to generate and stabilize the mutations. Solid mutants were inoculated with a suspension of $0.5 \times 10^{4}$ zoospores $\mathrm{ml}^{-1}$ of $P$. infestans. They were evaluated eight days later. Under field conditions, 116 mutants were assessed for resistance to late blight. Five controls were included, the resistant varieties INIAP-Santa Catalina, INIAP-Fripapa, and the susceptible controls Superchola, INIAP-Gabriela, and Uvilla. A partially balanced $11 \times 11$ lattice experimental design with three replications was used. Tukey test at 5\% was applied. The variables evaluated were area under the disease progress curve (AUDPC), based on 12 severity readings and the yield expressed in kilograms per plant. The individuals selected as solid mutants corresponded to $75 \%$ originating from apical buds (1319 mutants) and 69\% originating from axillary buds (2145 mutants). Eighty percent of mutants of axillary origin and $62 \%$ of apical origin showed a susceptibility reaction to P. infestans. The percentage of solid mutants that showed a resistance response was reduced in relation to the initial population, $20 \%$ of mutants were resistant of axillary origin and $38 \%$ of mutants of apical origin. For AUDPC, eight ranges were found. INIAP-Santa Catalina, INIAP-Fripapa, m67, m92, m39 were in the first ranges, with AUDPC lower than 2536.24. Regarding yield, three ranges were established. The $\mathrm{m} 42$ and $\mathrm{m} 65$ mutants were in the first range with $1.15 \mathrm{~kg}^{\text {plant }}{ }^{1}$. In the laboratory, 938 mutants with resistance P. infestans were selected. In the field, 18 mutants with resistance $P$. infestans were selected.
\end{abstract}

Keywords: Apical buds, Axillary buds, Explants, Severity, Yield.

\title{
INTRODUCCIÓN
}

La papa (Solanum tuberosum L.) es uno de los cultivos alimenticios más importantes del mundo después del arroz, trigo y maíz en términos de consumo humano [1]. Más de mil millones de personas en todo el mundo consumen papas y la producción mundial del cultivo superó los 368 millones de toneladas en 2018 [2].

En Ecuador, la producción nacional de papa en el 2018 fue de 269201 toneladas, cultivadas en 23974 hectáreas, con un rendimiento promedio de 16.28 t ha-1 [3]. Esta presentó una reducción del 29\% respecto al año 2017. Una de las causas se debió al efecto de las plagas que ocasionaron pérdidas en la producción del 13\% [4]. 
El cultivo de papa se ve afectado por factores bióticos (plagas y enfermedades) y abióticos (sequía, bajas temperaturas, calor, entre otros) que afectan la producción y calidad [5,6]. Una de las principales enfermedades que afecta al cultivo es el tizón tardío, causada por un Oomicete denominado Phytophthora infestans. Esta enfermedad puede causar pérdidas en el rendimiento superiores al 70\% [7,8,9].

La mayoría de variedades de papas cultivadas en Ecuador son susceptibles a tizón tardío y para combatir esta enfermedad una de las estrategias utilizada por los agricultores es el uso de fungicidas. Los agricultores ecuatorianos realizan hasta 23 aplicaciones en variedades susceptibles como es Superhola [10]. Otra alternativa es el mejoramiento genético mediante cruzamientos, selección clonal, selección por pedigrí, mutaciones inducidas entre los principales. La presente investigación tuvo como objetivo generar y seleccionar mutantes de papa con resistencia a P. infestans provenientes de la variedad Superchola, obtenidas mediante radiaciones $\operatorname{con}^{60} \mathrm{Co}$.

\section{MATERIALES Y MÉTODOS}

\section{Ubicación}

La investigación se efectuó en la Estación Experimental Santa Catalina del Instituto Nacional de Investigaciones Agropecuarias (INIAP), Cantón Mejía, Provincia de Pichincha, ubicada a una altitud de 3050 metros sobre el nivel del mar.

\section{Generación de mutantes}

A partir de plántulas de papa de la variedad Superchola cultivadas in vitro, se obtuvieron explantes de yemas apicales y axilares que fueron colocadas en 20 cajas Petri con agua desionizada estéril. Cada caja Petri contenía 50 explantes. En total se utilizaron 500 explantes provenientes de yemas axilares y 500 explantes de yemas apicales. El proceso de irradiación se efectuó con una fuente de ${ }^{60} \mathrm{Co}$, perteneciente al Departamento de Ciencias Nucleares de la Escuela Politécnica Nacional. Los explantes fueron sometidos a la dosis óptima determinada de 35 Gy para los explantes de yemas apicales y 30 Gy para los explantes de yemas axilares. Posteriormente, los explantes irradiados seleccionados se colocaron en un medio básico de cultivo Murashige \& Skoog (M\&) [11]. Se realizaron tres micropropagaciones sucesivas cada 45 días. Los mutantes sólidos (libres de quimeras), provenientes de yemas axilares y apicales identificados, fueron sembrados en frascos de vidrio de un diámetro de $10 \mathrm{~cm}$ con medio básico de cultivo M\&S. Cada frasco contenía cinco cortes de yemas separados de 2 a $3 \mathrm{~cm}$ entre cortes. Las condiciones de crecimiento in vitro fueron de $24^{\circ} \mathrm{C}, 16$ horas de luz.

\section{Inóculo de Phytophthora infestans}

Para las inoculaciones se utilizó un aislamiento de una raza compleja con 11 genes $R$ obtenida de la colección de aislamientos del INIAP. La activación, multiplicación, preparación y aplicación del inóculo de $P$. infestans se lo realizó de acuerdo a la metodología establecida por Gamboa, Pérez, Andrade-Piedra \& Forbes (2019) [12]. 


\section{Selección in vitro}

Los mutantes sólidos se inocularon cuando las plántulas presentaron entre tres a cuatro hojas completamente desarrolladas en frascos de vidrio (cinco plántulas por frasco), con una suspensión de $0.5 \times 10^{4}$ zoosporas $\mathrm{ml}^{-1}$ de inóculo de $P$. infestans. Después de ocho días de la inoculación, las plántulas fueron evaluadas por su respuesta al aislamiento para lo cual se utilizó la escala utilizada por Huang, Vleeshouwers, Visser \& Jacobsen (2005) [13] donde: 1 = lesión extendida con una esporulación masiva; 2 = poca extensión de la lesión o poca esporulación; 3 = lesión sin extensión con poca esporulación; 4 = lesión sin extensión, rastros de necrosis, sin esporulación; y 5 = sin síntomas.

\section{Evaluación en campo}

Se evaluaron 116 plantas mutantes que presentaron resistencia a tizón tardío en condiciones de laboratorio. Se utilizaron cinco variedades testigo: INIAP-Santa Catalina, INIAP-Fripapa, como resistentes, e INIAP-Gabriela, Superchola y Uvilla como susceptibles. El diseño experimental utilizado fue látice parcialmente balanceado $11 \times 11$ con tres repeticiones. Se utilizó la prueba de Tukey al 5\% para los tratamientos que presentaron diferencias estadísticas. Las variables evaluadas fueron severidad a tizón tardío expresada en valores de área bajo la curva de progreso de la enfermedad (ABCPE) y rendimiento en kilogramos por planta [14]. La severidad se evaluó cada 7 días a libre infección a partir de los 45 días después de la siembra, en total se realizaron 12 lecturas.

\section{RESULTADOS Y DISCUSIÓN}

\section{Mutantes sólidos}

Los individuos seleccionados como mutantes sólidos, correspondieron al 75\% originados de yemas apicales (1319 mutantes), mientras que un $25 \%$ fueron deformes (434 mutantes); y $69 \%$ seleccionados de yemas axilares (2145 mutantes), con 31\% de deformes (976 mutantes). En total se generaron 3464 mutantes sólidos y 1410 mutantes deformes. Las yemas apicales y axilares provenientes de Superchola in vitro irradiadas con fuente ${ }^{60} \mathrm{Co}$ generaron una población de mutantes sólidos. Bado et al., (2016) [15] menciona que este método de cultivos in vitro de papa irradiados con una fuente de ${ }^{60} \mathrm{Co}$ es una alternativa eficaz para generar variación genética.

\section{Reacción a Phytophthora infestans de mutantes in vitro}

Se evaluaron 1319 mutantes sólidos provenientes de yemas apicales. Se identificó que el 38\% presentaron una respuesta de resistencia a P. infestans ubicándose en la escala 4 y 5 con 316 y 182 mutantes, respectivamente; mientras que el $62 \%$ de los mutantes mostraron una reacción de susceptibilidad ubicándose en la escala 1 y 2, con 451 y 370 mutantes, respectivamente. Los mutantes sólidos provenientes de yemas axilares evaluados fueron 2145, de los cuales el $20 \%$ mostraron una respuesta de resistencia a $P$. infestans y se ubicaron en la escala 4 y 5 , con 179 y 261 mutantes, respectivamente. El 80\% de las yemas axilares mostraron susceptibilidad y se ubicaron en la escala 1 y 2, con 1044 y 1482 mutantes, respectivamente. En total, se evaluaron 3464 mutantes sólidos provenientes de yemas apicales y axilares y solo el 27.08\% 
(938 mutantes) presentaron resistencia a P. infestans. Gosal et al., (2001) [16] en su evaluación in vitro de mutantes provenientes de dos variedades presentaron valores similares con $42 \%$ y $36 \%$ de mutantes con resistencia a P. infestans, mientras Al-Safadi \& Arabi (2003) [5] en su evaluación in vitro a $P$. infestans en mutantes provenientes de tres variedades obtuvo menos del 5\% de mutantes con resistencia a tizón tardío.

El análisis de la varianza para ABCPE y rendimiento por planta (RP) estableció diferencias significativas al $1 \%$ de probabilidad. El promedio general fue de 3261.31 y 0.60 para ABCPE y kg planta ${ }^{-1}$, respectivamente. El coeficiente de variación (CV) fue de 15.92\% y 45.60\% para ABCPE y para RP, respectivamente (Tabla 1).

Tabla 1. Análisis de varianza para ABCPE y RP en la evaluación de mutantes con resistencia a P. infestans) en la variedad Superchola, EESC, Ecuador.

\begin{tabular}{|l|c|c|c|}
\multirow{2}{*}{ Fuente de Variación } & \multirow{2}{*}{ GL } & \multicolumn{2}{|c}{ Cuadrados Medios } \\
\cline { 2 - 4 } & & ABCPE & RP \\
\hline Total & 362 & - & - \\
\hline Repetición & 2 & $5.82^{\text {ns }}$ & $38.96^{\text {ns }}$ \\
\hline Mutantes & 120 & $2.51^{* *}$ & $2.10^{* * *}$ \\
\hline Bloque ajustado & 10 & $2.01^{* *}$ & $4.59^{* * *}$ \\
\hline Error Intrabloque & 132 & $2.52^{* *}$ & $2.84^{* *}$ \\
\hline Error & 230 & & \\
\hline CV (\%) & & 15.92 & 45.60 \\
\hline PG & & 3261.31 & 0.60 \\
\hline
\end{tabular}

${ }^{* *}$ Significativa al 1\%"ns no significativo; $\mathrm{CV}=$ coeficiente de variación; $\mathrm{PG}=$ promedio general; $\mathrm{ABCPE}=$ área bajo la curva de progreso de la enfermedad; $\mathrm{RP}=$ rendimiento por planta $(\mathrm{kg})$.

\section{Área bajo la curva de progreso de la enfermedad (ABCPE)}

Al realizar la prueba de Tukey al 5\% para ABCPE se encontraron ocho rangos. Las variedades INIAP-Santa Catalina, INIAP-Fripapa y los mutantes m67, m92, m39 se ubicaron en los primeros tres rangos con valores menores a 2536.25 unidades de ABCPE; mientras que el mutante m75 se ubicó en el último rango con 4829.52 unidades de ABCPE (Tabla 2). Los tres mutantes (m67, m92, m39) mostraron la mayor resistencia a $P$. infestans lo que representan el $2.59 \%$ de los mutantes evaluados (116 mutantes). Kowalski \& Cassells (1999) [17] obtuvieron un porcentaje mayor de mutantes con resistencia a tizón tardío (7\%) provenientes de una variedad comercial. Dentro de las variedades testigo, Superchola y Uvilla fueron los más susceptibles a P. infestans con valores de 3716.86 y 4237.14 unidades de ABCPE, respectivamente. A pesar de que existió variación en la respuesta de los mutantes a $P$. infestans, no se encontraron mutantes con mayor resistencia a la mostrada por la variedad testigo INIAP-Santa Catalina.

\section{Rendimiento por planta (RP)}

Al realizar la prueba de Tukey al 5\% para RP se establecieron tres rangos, los mutantes m42, y m65 se ubicaron en el primero con $1.15 \mathrm{~kg}^{\text {planta-1. }}$. La variedad INIAP-Santa Catalina y el 
mutante m49 se ubicaron en el segundo con rendimientos entre 1.09 y $1.12 \mathrm{~kg}$ planta ${ }^{-1}$, respectivamente; mientras que los mutantes $\mathrm{m} 115, \mathrm{~m} 37, \mathrm{~m} 69$ y $\mathrm{m} 24$ se encontraron en el último rango con rendimientos inferiores a $0.24 \mathrm{~kg}_{\text {planta-1 }}{ }^{-1}$. Las variedades susceptibles Superchola, Uvilla e INIAP-Gabriela presentaron rendimientos de $0.85,0.40$ y $0.30 \mathrm{~kg}^{\text {planta-1}}{ }^{-1}$, respectivamente (Tabla 2). Al comparar el rendimiento de los mutantes m42, m65 y m49 con Superchola (variedad de la que provienen los mutantes), se observó un incremento en el rendimiento de entre el 24.11\% al 26.09\%. Resultados similares lo obtuvo Salomón, González, Castillo \& Varela (2017) [18] al evaluar mutantes provenientes de una variedad irradiada con fuente ${ }^{60} \mathrm{Co}$ (30 Gy), que reportó un incremento promedio del rendimiento por planta de $43.86 \%$ comparado con la variedad de origen de los mutantes. Se identificó mutantes con rendimientos similares o superiores a las variedades resistentes, los cuales tienen potencialidad para seguir sus evaluaciones dentro del esquema de mejoramiento del Programa Nacional de Raíces y Tubérculos, rubro papa, del INIAP establecido en Cuesta, Rivadeneira \& Monteros (2015) [14].

Tabla 2. Promedio y prueba de Tukey al $5 \%$ para ABCPE, RP en materiales mutantes y variedades testigo en la EESC, Ecuador.

\begin{tabular}{|c|c|c|c|c|c|c|c|c|}
\hline Genotipos & ABCPE & $\mathbf{R P}$ & Genotipos & ABCPE & $\mathbf{R P}$ & Genotipos & ABCPE & RP \\
\hline I-Sta. Catalina & 1638.88a & $1.09 a b$ & m32 & $3084.74 a-g$ & $0.57 a b c$ & m116 & $3401.96 b-h$ & $0.38 a b c$ \\
\hline I-Fripapa & $2304.69 a b$ & $0.72 a b c$ & m63 & $3086.54 a-9$ & $0.63 a b c$ & $\mathrm{~m} 70$ & $3404.18 b-h$ & $0.52 a b c$ \\
\hline m67 & $2373.31 a b c$ & $0.70 a b c$ & m107 & $3089.55 \mathrm{~d}-\mathrm{g}$ & $0.50 a b c$ & $\mathrm{~m} 1$ & $3436.73 b-h$ & $0.72 a b c$ \\
\hline m92 & $2522.07 \mathrm{a}-\mathrm{d}$ & $0.73 a b c$ & m52 & $3097.66 \mathrm{a}-\mathrm{g}$ & $0.32 a b c$ & m74 & $3443.41 b-h$ & $0.72 a b c$ \\
\hline m39 & $2536.24 \mathrm{~d}-\mathrm{d}$ & $1.02 \mathrm{abc}$ & m103 & $3110.44 \mathrm{a}-\mathrm{g}$ & $0.56 a b c$ & m35 & $3450.19 b-h$ & $0.70 a b c$ \\
\hline m57 & 2583.76 a-e & $0.30 a b c$ & $\mathrm{~m} 110$ & $3113.40 \mathrm{a}-\mathrm{g}$ & $0.73 a b c$ & m51 & $3466.42 b-h$ & $0.85 a b c$ \\
\hline m113 & $2610.05 a-f$ & $1.00 a b c$ & m16 & $3117.79 a-g$ & $0.50 a b c$ & m104 & $3479.71 b-h$ & $0.60 a b c$ \\
\hline $\mathrm{m} 25$ & $2626.14 a-f$ & $0.82 a b c$ & m86 & $3132.67 \mathrm{~d}-\mathrm{g}$ & $0.72 a b c$ & m80 & $3501.92 \mathrm{~b}-\mathrm{h}$ & $0.52 a b c$ \\
\hline m8 & 2661.35 a-f & $0.45 a b c$ & m41 & $3141.97 \mathrm{~d}-\mathrm{g}$ & $0.58 a b c$ & m17 & $3508.03 b-h$ & $0.37 a b c$ \\
\hline m58 & $2692.00 \mathrm{a}-\mathrm{f}$ & $0.82 a b c$ & m81 & $3148.38 a-h$ & $0.27 b c$ & m56 & $3510.17 b-h$ & $0.78 a b c$ \\
\hline m117 & $2709.69 a-f$ & $0.27 b c$ & $\mathrm{~m} 47$ & $3148.99 a-h$ & $0.90 a b c$ & m53 & 3531.11 b-h & $0.53 a b c$ \\
\hline m77 & $2716.60 \mathrm{a}-\mathrm{f}$ & $0.40 a b c$ & m96 & $3171.58 \mathrm{a}-\mathrm{h}$ & $0.79 a b c$ & $\mathrm{~m} 48$ & $3568.36 \mathrm{~b}-\mathrm{h}$ & $0.78 a b c$ \\
\hline m11 & 2723.96 a-f & $0.72 a b c$ & m4 & $3176.69 a-h$ & $0.57 a b c$ & m15 & $3569.84 b-h$ & $0.28 a b c$ \\
\hline m89 & $2736.12 \mathrm{a}-\mathrm{f}$ & $1.05 a b c$ & m38 & $3178.65 a-h$ & $0.30 a b c$ & m98 & $3631.96 \mathrm{~b}-\mathrm{h}$ & $0.63 a b c$ \\
\hline m112 & $2757.30 \mathrm{a}-\mathrm{f}$ & $0.82 a b c$ & m65 & $3200.22 a-h$ & $1.15 a$ & m105 & $3642.09 b-h$ & $0.52 a b c$ \\
\hline m49 & 2757.30 a-f & $1.12 a b$ & m30 & 3200.61 a-h & $0.42 a b c$ & $\mathrm{~m} 40$ & $3654.79 b-h$ & $0.97 a b c$ \\
\hline m13 & $2778.92 \mathrm{a}-\mathrm{f}$ & $0.81 a b c$ & m62 & 3201.52a-h & $0.62 a b c$ & m19 & $3703.32 b-h$ & $0.32 a b c$ \\
\hline m111 & 2790.44 a-f & $0.55 a b c$ & $\mathrm{~m} 12$ & $3226.16 a-h$ & $0.47 a b c$ & m85 & $3712.00 \mathrm{~b}-\mathrm{h}$ & $0.37 a b c$ \\
\hline $\mathrm{m} 43$ & $2798.96 \mathrm{a}-\mathrm{f}$ & $0.62 a b c$ & m9 & $3229.18 a-h$ & $0.47 a b c$ & m109 & $3716.49 b-h$ & $0.28 a b c$ \\
\hline m55 & 2806.70 a $-f$ & $1.03 \mathrm{abc}$ & |-Gabriela & $3235.66 a-h$ & $0.30 a b c$ & Superchola & $3716.86 \mathrm{~b}-\mathrm{h}$ & $0.85 a b c$ \\
\hline $\mathrm{m} 120$ & 2809.37 d-f & $0.65 a b c$ & $\mathrm{~m} 26$ & $3248.74 a-h$ & $0.78 a b c$ & m6 & $3717.94 \mathrm{~b}-\mathrm{h}$ & $0.58 \mathrm{abc}$ \\
\hline $\mathrm{m} 73$ & 2814.27 a-f & $0.75 a b c$ & m66 & 3252.00 a-h & $0.70 a b c$ & $\mathrm{~m} 28$ & $3731.48 b-h$ & $0.41 a b c$ \\
\hline m7 & 2830.67 a-f & $0.80 a b c$ & m45 & $3260.23 a-h$ & $0.98 \mathrm{abc}$ & m95 & $3749.01 b-h$ & $0.75 a b c$ \\
\hline m100 & 2885.27 a-f & $0.37 a b c$ & m64 & $3263.34 a-h$ & $0.78 a b c$ & m68 & $3761.29 b-h$ & $0.38 a b c$ \\
\hline m82 & 2889.57 a-f & $0.60 a b c$ & m44 & $3263.46 a-h$ & $0.60 a b c$ & m106 & $3762.99 \mathrm{~b}-\mathrm{h}$ & $0.63 a b c$ \\
\hline m83 & $2915.43 \mathrm{a}-\mathrm{f}$ & $0.65 a b c$ & m37 & $3275.50 a-h$ & $0.22 c$ & $\mathrm{~m} 76$ & $3778.66 \mathrm{~b}-\mathrm{h}$ & $0.47 a b c$ \\
\hline $\mathrm{m} 46$ & $2933.25 \mathrm{a}-\mathrm{f}$ & $0.77 a b c$ & m36 & $3286.74 a-h$ & $0.47 a b c$ & m18 & $3780.87 b-h$ & $0.27 b c$ \\
\hline
\end{tabular}




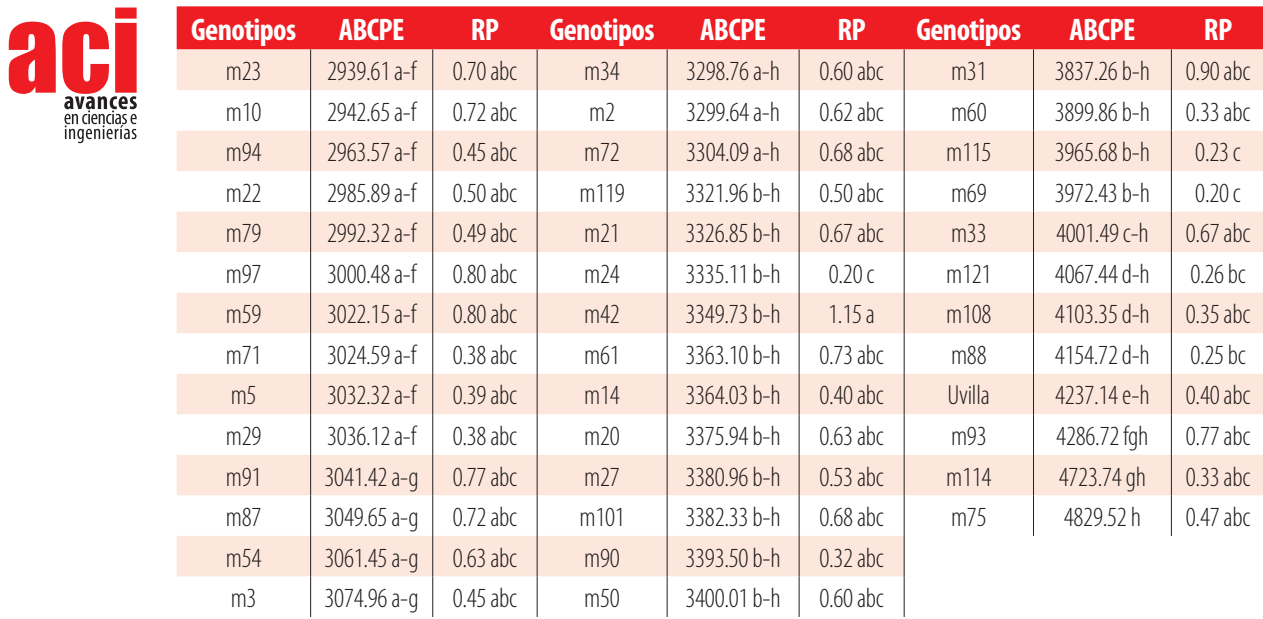

'Letras diferentes indican diferencias estadísticas según Tukey al 5\%; RP = rendimiento por planta; $\mathrm{ABCPE}=$ área bajo la curva de progreso de la enfermedad; $\mathrm{RP}=$ rendimiento por planta $(\mathrm{kg}) . \mathrm{l}=\mathrm{INIAP}$.

\section{CONCLUSIONES}

El uso de radiación con fuente ${ }^{60} \mathrm{Co}$ es una alternativa válida para generar variación para resistencia a tizón tardío. La evaluación de mutantes in vitro para P.infestans es una opción para una selección preliminar cuando existen grandes poblaciones. Las evaluaciones en campo permitieron identificar 18 mutantes que mostraron características de resistencia a P. infestans y buenos rendimientos.

\section{AGRADECIMIENTOS}

Esta investigación se ejecutó en el marco del proyecto del Organismo Internacional de Energía Atómica (OIEA) "Inducing Mutation in Agriculture with the Aid of Radiation". Un resumen de este trabajo se presentó en el II Simposio Latinoamericano de Aplicaciones Nucleares en la Agricultura realizado en marzo de 2020 en la ciudad de Quito, Ecuador.

Al Laboratorio de Tecnología de Radiaciones del Departamento de Ciencias Nucleares de la Escuela Politécnica Nacional por el apoyo y colaboración en los procesos de irradiación al material vegetal con la fuente de ${ }^{60} \mathrm{Co}$.

\section{CONTRIBUCIONES DE LOS AUTORES}

Jorge Rivadeneira y Xavier Cuesta concibieron la investigación; Roberto López y María Villavicencio desarrollaron la metodología, análisis estadístico y realizaron el trabajo de laboratorio y campo; Jorge Rivadeneira y Xavier Cuesta redactaron el manuscrito; Xavier Cuesta revisó críticamente el contenido del manuscrito. 


\section{REFERENCIAS}

[1] Zhang, S., Zheng, X., Reiter, R., Feng, S., Wang, S., Liu, S., Jin, L., Li, Z., Datla, R. \& Ren, M. (2017). Melatonin Attenuates Potato Late Blight by Disrupting Cell Growth, Stress Tolerance, Fungicide Susceptibility and Homeostasis of Gene Expression in Phytophthora infestans. Frontier in Plant Science. 8:1993. Doi: 10.3389/fpls.2017.01993

[2] FAO. (2020). FAOSTAT Statistics Database 2018. Consultado abril del 2020. Disponible en: [http://www.fao.org/ faostat/en/\#data].

[3] Instituto Nacional de estadística y censos - INEC. (2019). Encuesta y superficie y producción agropecuaria continua. https://www.ecuadorencifras.gob.ec/estadisticas-agropecuarias-2/

[4] Ministerio de Agricultura y Ganadería - MAG. (2019). "Sistema de Información Pública Agropecuaria. http://sipa. agricultura.gob.ec/index.php/papa

[5] Al-Safadi, B. \& Arabi, M. (2003). In vitro induction, isolation and selection of potato mutants resistant to late blight. J.Genet \& Breed., 57:364-359.

[6] Gabriel, J., Ruiz, I. \& Cuesta, X. (2018). Ampliando la frontera agrícola de la papa (Solanum tuberosum L.) para disminuir los efectos del cambio climático. Universidad y Sociedades. 10(1):46-51.

[7] Sedláková, V., Dejmalová, J., Hausvater, E., Sedlák, P., Doležal, P. \& Mazaková, J. (2011). Effect of Phytophthora infestans on potato yield in dependence on variety characteristics and fungicide control. Plant, Soil and Enviroment. 57:486-491.

[8] Fry, W., Birch, P., Judelson, H., Grünwald, N., Danies, G., Everts, K., Gevens, A., Gugino, B., Johnson, D., Johnson, S. et al. (2015). Five reasons to consider Phytophthora infestans a Reemerging Pathogen. Phytopathology, 105: 966-981.

[9] Lenman, M., Ali, A., Mühlenbock, P., Carlson-Nilsson, U., Liljeroth, E., Champouret, N., Vleeshouwers, V. \& Andreasson, E. (2015). Effector-driven marker development and cloning of resistance genes against Phytophthora infestans in potato breeding clone SW93-1015. Theor App/ Genet. 129: 105-115. Doi: https://doi.org/10.1007/s00122-015-2613

[10] Unda, J., Suquillo, J., Sevillano, C., Pumisacho, M., Ochoa,J. \& Barrera, V. (2013). Diagnóstico del manejo de Tizón tardío en la provincia del Carchi, Ecuador. Riobamba, Ecuador: V Congreso Ecuatoriano de la papa.

[11] Murashige, T. \& Skoog, F. (1962). A revised medium for rapid growth and biossays with tobacco cultures. Physiologia Plantarum, (15), 473-497.

[12] Gamboa, S., Pérez, W., Andrade-Piedra, J. \& Forbes, G. (2019). Laboratory manual for Phytophthora infestans work at CIP. Lima, Perú: International Potato Center. 78pp.

[13] Huang, S., Vleeshouwers, V., Visser, R. \& Jacobsen, E. (2005). An accurate in vitro assay for high-throughput disease testing of Phytophthora infestans in potato. Plant Dis. 89:1263-1267.

[14] Cuesta, X., Rivadeneira, R. \& Monteros, C. (2015). Mejoramiento genético de papa: conceptos, procedimientos, metodologías y protocolos. Quito, Ecuador. Insituto Nacional de Investigaciones Agropecuarias. $62 \mathrm{p}$.

[15] Bado, S.,. Rafiri, M., El-Achouri, K., Sapey, E., Nielen, S., Ghanim, A., Forster, B. \& Laimer, M. (2016). In vitro methods for mutation induction in potato (Solanum tuberosum L.). African Journal of Biotechnology, 15, 39.

[16] Gosal, S.S., Das, A., Gopal, J., Minocha, J.L., Chopra, H.R., \& Dhaliwal, H.S. (2001). In vitro induction of variability through radiation for late blight resistance and heat tolerance in potato (IAEA-TECDOC--1227). International Atomic Energy Agency (IAEA)

[17] Kowalski, B. \& Cassells, A. (1999). Mutation breeding for yield and Phytphthora infestans (Mont.) de Bary folia resistance in potato (Solanum tuberosum L: cv Golden Wonder) using computerized image analysis in selection. Potato Research. 42:121-130.

[18] Salomón, J., González, M., Castillo, J. \& Varela, M. (2017). Comportamiento de "Barna", cultivar de papa (Solanum tuberosum L.) ante diferentes dosis de rayos gamma de Fuente cobalto 60. Cultivos Tropicales, 38(4):127-130. 